\title{
Ni(111) Thin Layers Recrystallization Studied by SEM, EBSD and AFM
}

\author{
Dominika Teklinska ${ }^{1}$, Iwona Jozwik ${ }^{1,2}$ and Piotr Knyps ${ }^{1}$ \\ ${ }^{1 .}$ Institute of Electronic Materials Technology, Warsaw, Poland \\ 2. National Centre for Nuclear Research, NOMATEN Centre of Excellence, Swierk-Otwock, Poland
}

Hexagonal boron nitride (h-BN), sometimes called "white graphene" is a new type of wide bandgap nanomaterials with excellent properties and potential applications [1-3]. It can be grown by MOCVD technique over various substrates, but the epitaxial growth of any material requires that the crystallographic structure of deposited material need to be fitted as much as possible to the substrate to obtain high quality structure. Fulfilling this criterion ensures monocrystalline structure and low dislocations density in the epitaxially grown layer. In case of h-BN epitaxial layers, the best substrate from the point of view of crystal structure match is monocrystalline $\mathrm{Ni}(111)$. However due to very high price and limited dimensions of single crystalline Ni substrates, their application for epitaxial growth of $\mathrm{h}-\mathrm{BN}$ is not reasonable. The substrates used in the electronic industry must be wide in diameter scaling up to a few inches and available at relatively low price. To overcome the problem of the substrate size in case of h-BN epitaxial growth, the polycrystalline $\mathrm{Ni}$ thin layers may be deposited on the appropriate substrate, which crystallographic lattice will fit to $\mathrm{Ni}(111)$, and subsequently recrystallized forming a thin single crystalline layer oriented along [111]. The best choice for carrying substrate is $\mathrm{Al}_{2} \mathrm{O}_{3}(0001)$ which is available commercially at relatively low prices and may be used in re-cycled mode. The main aim of this work was the elaboration of recrystallization technology of polycrystalline nickel layers sputtered on sapphire substrate for obtaining monocrystalline nickel layer (111), which can be widely used as the template substrate for h-BN epitaxial growth.

Polycrystalline $250 \mathrm{~nm}$-thick Ni layers were sputtered on sapphire substrates by means of e-beam sputtering (Kurt Lesker E-Beam). In the next step the samples were subjected to series of processes aiming to optimization of Ni recrystallization conditions depending on $\mathrm{T}, \mathrm{p}, \mathrm{t}$ or atmosphere $\left(\mathrm{Ar}\right.$ or $\left.\mathrm{H}_{2}\right)$ using CVD reactor annealing. The samples were characterized by SEM, AFM and EBSD techniques.

The main achievement of the experimental part was the optimization of conditions of recrystallization of polycrystalline nickel sputtered over the $\mathrm{Al}_{2} \mathrm{O}_{3}$ (0001) into preferentially (111) oriented thin Ni layer. The studies performed by EBSD (further confirmed by XRD) have clearly shown that the obtained layer was single crystalline, however two typical crystallographic effects have been observed for recrystallized layers: the presence of mosaic twisted structure and low-angle boundaries.

Figure 1 presents the SEM images registered in BSE mode. The low-angle grain boundaries in channelling contrast in BSE mode were observed when the sample was tilted by a few tenths of a degree. The domains were in visible in SEM images (both SE and BSE). The results of EBSD measurements (Fig. 2) clearly show single crystalline nature of the recrystallized Ni layer, as well as reveal the presence of the mosaic twisted structure. The twist angle between the local domains has been measured to be $60 \mathrm{deg}$. The low-angle boundaries visible in the channelling contrast were also registered in MO (misorientation) representation of analyzed EBSD maps. To make sure that the effect is related to the Ni layer surface topography, the sample was investigated using AFM. The results confirming the findings are presented in Fig. 3. [4]. 


\section{References:}

[1] M H Khan et al, Materials Today 20 (2017), p. 611.

[2] S Sundaramet al, J. Cryst. Growth 509 (2019), p. 40.

[3] J Wang et al, Materials Today Physics 2 (2017), p.6.

[4] This work was partially sponsored by The Polish Ministry of Science and Higher Education, in the frame of the research project entitled „Recrystallization of nickel layers on the sapphire substrates” held at the Institute of Electronic Materials Technology, Warsaw, Poland.
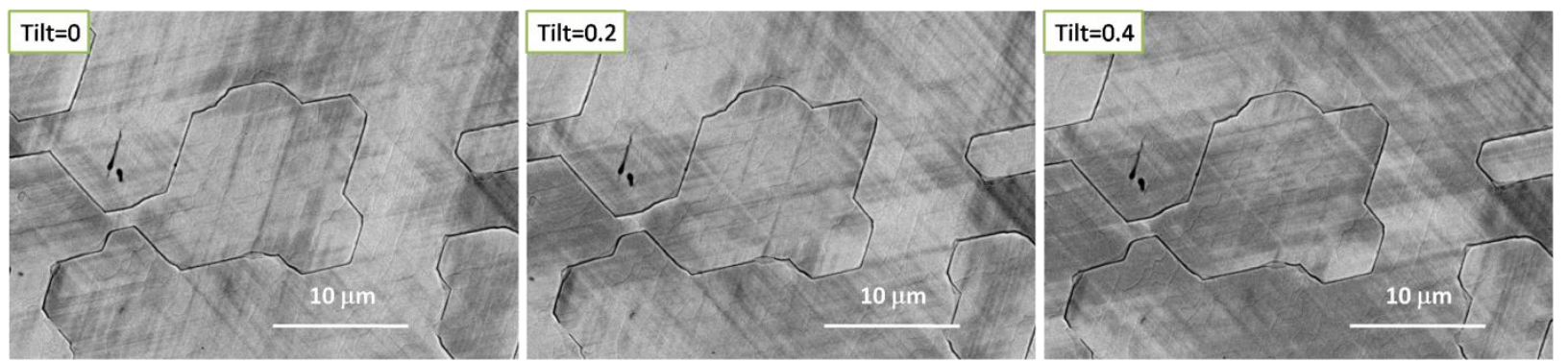

Figure 1.SEM BSE images presenting small angle grain boundaries visible in channeling contrast; mosaic domains in recrystallized $\mathrm{Ni}$ are also visible.
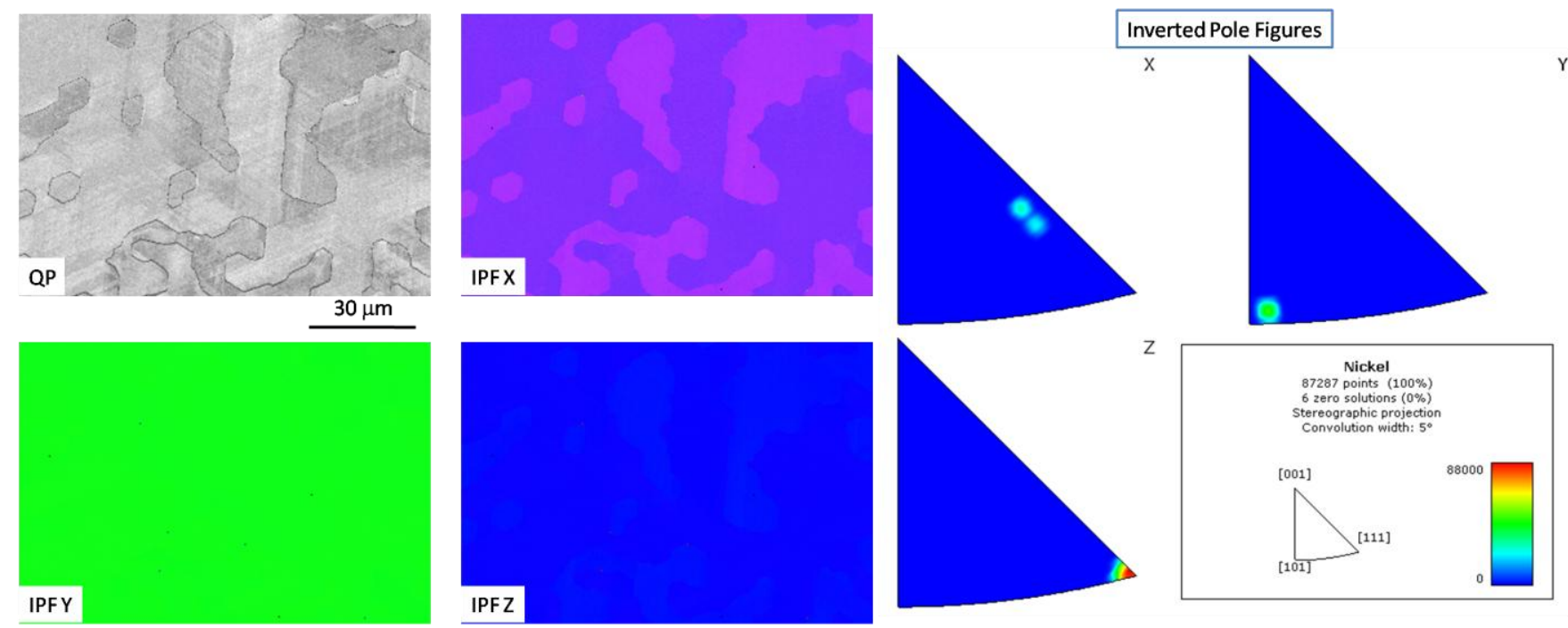

Figure 2. EBSD maps of $250 \mathrm{~nm}$ thick recrystallized Ni layer; mosaic tilt structure is visible in QP and IPF X maps; IPF X confirm the 60deg misorientation between twisted domains.

a)
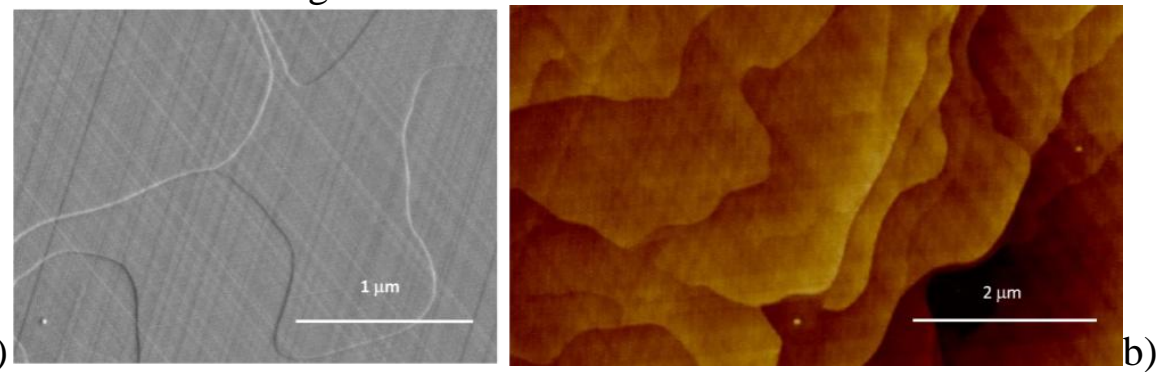

Figure 3. SEM (a) and AFM (b) images of selected sample registered in topography mode; the topography related to the presence of low-angle boundaries is clearly presented. 